Ann. Biol. anim. Bioch. Biophys., 1978, 18 (1), 81-85.

\title{
Recherche d'un mécanisme de transfert placentaire du calcium chez la rate gestante
}

\author{
par Christiane LEGRAND, A. RABIE, P. BESANÇON \\ Laborataires de Physiclogie Comparée et de Physiologie de la Nutrifion \\ Université de Montpellier II, 34060 Montpellier Cedex.
}

Summary. A suggested mechanism of placental calcium transfer in pregnant rat.

A calcium-binding protein and/or a Ca-stimulated ATPase may be involved in the placental transfer of calcium in the rat. At 20 days of gestation, Sephadex chromatography of placental extract suggested the existence of a calcium-binding protein $(M$. W. $\sim 20000$ ). At 18 days of gestation, Ca-ATPase activity, as well as placental calcium transfer, increased. Neither hypothyroidism nor parathyroidectomy of the mother modified Ca-ATPase activity.

Au cours de la gestation, le calcium, nécessaire à l'édification des tissus osseux du fœetus, franchit le placenta par un mécanisme de transport actif. Le passage transplacentaire augmente sensiblement vers la fin de la gestation, en même temps qu'apparaît une légère hypercalcémie fotale, à partir du $18^{\mathrm{e}}$ jour de gestation chez le Rat. Le mécanisme de transport n'est pas élucidé. S'agit-il d'un système faisant intervenir une protéine fixant spécifiquement le calcium (calcium binding protein, CaBP), que selon Kretsinger (1976), il serait possible de mettre en évidence chaque fois qu'il y a transport actif de calcium ? Le placenta n'a jamais été étudié à cet égard. S'agit-il aussi d'un système impliquant l'intervention d'une ATPase Ca-dépendante, intervenant au niveau des membranes? Shami et Radde (1971) ont en effet montré l'existence d'une Ca-ATPase dans les membranes plasmiques du placenta de Cobaye.

Nous avons tenté d'identifier un système de transport dans le placenta de rate - CaBP et/ou Ca-ATPase - et avons étudié l'évolution de l'activité ATPasique en fonction du stade de gestation ainsi que lors de perturbations de l'équilibre endocrinien de la mère : hypothyroïdie ou parathyroïdectomie.

\section{Matériel et méthodes.}

Recherche d'une CaBP placentaire. Les rates, provenant de l'élevage du laboratoire, sont sacrifiées au 21e jour de gestation. Les placentas sont lavés $(\mathrm{NaCl} 9$ p. 1000 à $4^{\circ} \mathrm{C}$ ), homogénéisés en milieu tamponné (TRIS $0,1 \mathrm{M}$ à $20 \mathrm{p} .100 \mathrm{P} / \mathrm{V}, \mathrm{pH} 7,4$, à $4^{\circ} \mathrm{C}$, durant $3 \mathrm{mn}$ ) ; les homogénats sont centrifugés $(38000 \mathrm{~g}, 20 \mathrm{mn})$. Le surnageant est élué sur des colonnes calibrées de Sephadex G 25, G 75 ou G 100 . Sur chacune des 
fractions la fixation du calcium radioactif $\left({ }^{45} \mathrm{CaCl}_{2}\right)$ est déterminée par compétition avec une résine Chelex à $\mathrm{pH} 7,4$. La résine Chelex est éliminée par centrifugation $(4500 \mathrm{t} / \mathrm{mn}, 10 \mathrm{mn}$ ) et la radioactivité mesurée dans le surnageant (Wasserman $\epsilon \dagger$ Taylor, 1966).

Dosage de l'activité ATPasique Ca-dépendante placentaire. La méthode est empruntée à Whittaker ef Barker (1972). Le placenta est broyé dans une solution de sucrose $0,32 \mathrm{M} .100 \mu \mathrm{l}$ de broyat sont incubés à $37^{\circ} \mathrm{C}$ en présence de $0,5 \mathrm{mM}$ d'ATP, $0,5 \mathrm{mM}$ d'ions $\mathrm{Ca}^{++}, 0,5 \mathrm{mM}$ d'ions $\mathrm{Mg}^{++}$ou de l'un d'eux seulement. L'activité ATPasique est mesurée par la libération de phosphate inorganique (Pi). Nous avons vérifié d'une part que l'activité ATPasique mesurée n'est pas $\mathrm{Na} / \mathrm{K}$-dépendante, qu'elle est stimulée par les ions $\mathrm{Ca}^{++}$et/ou $\mathrm{Mg}^{++}$, d'autre part que l'activité ATPasique du sang maternel ou foetal, qui peut contaminer le broyat placentaire, est négligeable par rapport aux valeurs obtenues dans le placenta.

Réalisation de l'hypothyroïdie ef de la parathyroïdectomie. Des rates sont rendues hypothyroidiennes par administration orale de $50 \mathrm{mg}$ de propylthiouracyle par jour durant 6 jours avant le sacrifice. D'autres rates sont parathyroïdectomisées au $7 \mathrm{e}$ ou au $14^{\mathrm{e}}$ jour de gestation et sacrifiées au $21^{\mathrm{e}}$ jour.

\section{Résultats.}

Fixation de calcium sur une CaBP placentaire (fig. 1). Après séparation chromatographique des protéines solubles du surnageant de placenta, sur une colonne de

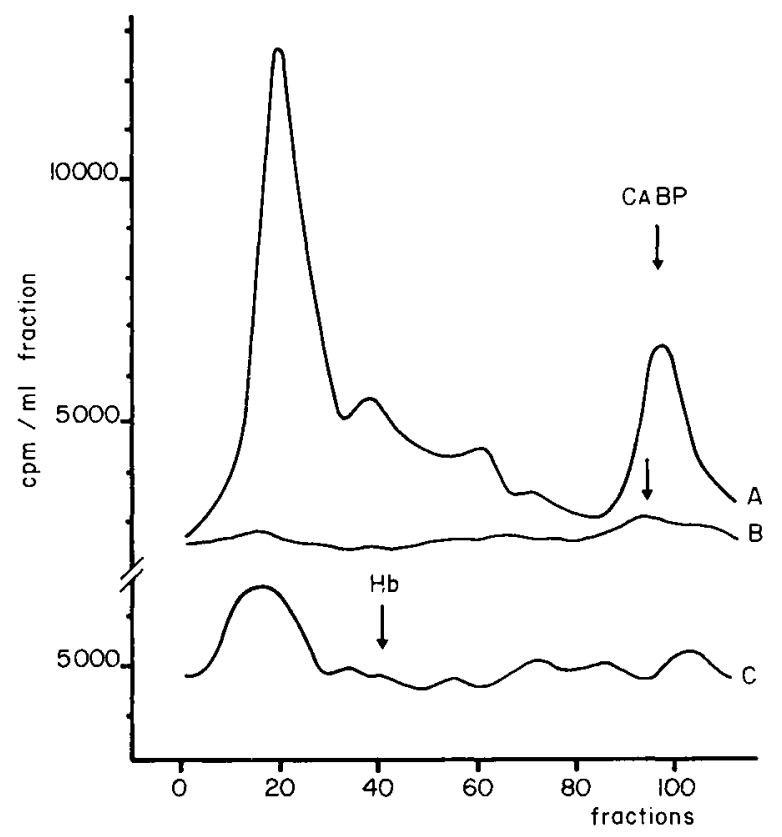

FIG. 1. - Fixation du calcium sur les protéines sanguines et placentaires (Sephadex G 100.)

A : Protéines placentaires ; B : Protéines placentaires, après chauffage ; $C$ : Protéines sanguines. 
Sephadex G 25 , on observe une fixation systématique de calcium-45, qui semble spécifique des protéines placentaires et non des protéines sanguines.

La chromatographie sur des colonnes de Sephadex G 75 ou G 100 permet de séparer plusieurs fractions protéiques capables de fixer le calcium : une fraction de faible masse molaire, voisine de 20000 , ainsi que des protéines à masse molaire élevée, autres que l'hémoglobine. L'action de la chaleur, par incubation à $60^{\circ} \mathrm{C}$ durant $10 \mathrm{mn}$, fait disparaître la fixation de ${ }^{45} \mathrm{Ca}$ sur les grosses protéines. La comparaison avec du sang maternel, prélevé au $21 \mathrm{e}$ jour de gestation, permet de vérifier que les protéines placentaires qui fixent le calcium sont différentes des protéines sanguines : celles-ci ne sont pas éluées au même volume d'exclusion. La fixation de calcium sur de petites protéines solubles du placenta n'est cependant pas systématique, bien que les résultats obtenus, lorsque la fixation est mise en évidence, soient très reproductibles (volume d'exclusion, poids moléculaire). Des essais de marquage in vivo donnent des résultats similaires, lorsqu'on injecte du calcium-45 à la mère entre 5 et $15 \mathrm{mn}$ avant le sacrifice. On observe cependant un marquage très diffus au niveau des protéines sanguines maternelles ef fœtales.
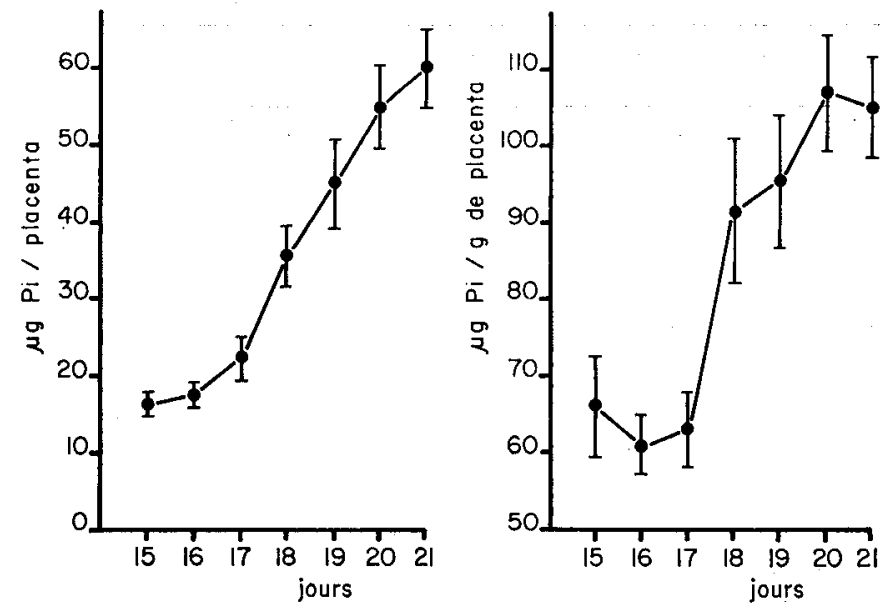

FIG. 2. - Activité ATPasique Ca-dépendante placentaire.

Activité Ca-ATPasique placentaire (fig. 2). L'activité Ca-ATPasique placentaire mesurée entre les $15^{\mathrm{e}}$ ef $21^{\mathrm{e}}$ jours de gestation augmente toujours avec une accélération rapide vers le $18 \mathrm{e}$ jour. Cette évolution n'est pas seulement liée à la croissance pondérale du placenta puisque l'augmentation reste significative si on rapporte l'activité au poids de placenta. Cette augmentation correspond à l'apparition des points d'ossification chez le fœetus et à l'installation de l'hypercalcémie fœtale par rapport à la calcémie maternelle (Pic, 1969). L'activité ATPasique augmente encore jusqu'au $21^{\mathrm{e}}$ jour, âge auquel la calcémie fœtale atteint $111 \pm 3 \mathrm{mg} / \mathrm{l}$, pour une calcémie maternelle de $98 \pm 7 \mathrm{mg} / \mathrm{l}$.

Activité ATPasique placentaire chez les rates hypothyroïdiennes ou parathyroïdectomisées (łabl. 1). Chez les rates hypothyroïdiennes l'activité ATPasique placentaire, 
mesurée aux $18^{\mathrm{e}}$ et $21^{\mathrm{e}}$ jours de gestation, n'est pas significativement différente des témoins, l'augmentation de l'activité ATPasique au 18 jour semblant maintenue. La calcémie maternelle des rates parathyroïdectomisées est abaissée à $49 \pm 11 \mathrm{mg} / \mathrm{l}$, tandis que la calcémie fœtale passe à $96 \pm 11 \mathrm{mg} / \mathrm{l}$. Le poids des fœtus est également diminué de façon significative, de $4,7 \pm 0,3 \mathrm{~g}$ à $3,7 \pm 0,7 \mathrm{~g}$. L'activité Ca-ATPasique par placenta est très légèrement diminuée de façon non significative, que la parathyroîdectomie ait lieu à 7 ou à 14 jours.

TABLEAU 1

Activité ATPasique placentaire chez les rafes hypothyroïdiennes (PTU) ou parathyroïdectomisées (PTX)

\begin{tabular}{|c|c|c|c|}
\hline & $\begin{array}{l}\text { date du } \\
\text { sacrifice }\end{array}$ & $\begin{array}{l}\text { activité C } \\
\mu \mathrm{g} \mathrm{Pi} / \text { placenta }\end{array}$ & $\begin{array}{l}\text {-ATPasique } \\
\mu \mathrm{g} \mathrm{Pi} / \mathrm{g} \text { placenta }\end{array}$ \\
\hline Témoins $\ldots \ldots \ldots \ldots \ldots$ & $\begin{array}{l}17 j \\
18 j \\
21 j\end{array}$ & $\begin{array}{l}22 \pm 3 \\
35 \pm 10 \\
63 \pm 5\end{array}$ & $\begin{array}{r}63 \pm 4 \\
92 \pm 9 \\
110 \pm 8\end{array}$ \\
\hline PTU $\ldots \ldots \ldots \ldots \ldots \ldots$ & $\begin{array}{l}18 j \\
21 j\end{array}$ & $\begin{array}{l}38 \pm 10 \\
61 \pm 8\end{array}$ & $\begin{array}{r}93 \pm 27 \\
119 \pm 10\end{array}$ \\
\hline $\begin{array}{l}\text { PTX } 7 \text { j } \ldots \ldots \ldots \ldots \ldots \ldots \\
\text { PTX } 14 \text { j } \ldots \ldots \ldots \ldots \ldots \ldots \\
\text { Pseudo opérées } \ldots \ldots \ldots \ldots\end{array}$ & $\begin{array}{l}21 j \\
21 j \\
21 j\end{array}$ & $\begin{array}{l}59 \pm 21 \\
56 \pm 14 \\
74 \pm 19\end{array}$ & $\begin{array}{l}109 \pm 34 \\
132 \pm 35 \\
130 \pm 29\end{array}$ \\
\hline
\end{tabular}

\section{Discussion ef Conclusion.}

L'existence d'une protéine fixatrice du calcium dans le placenta est probable, bien qu'il ne soit pas possible de la mettre systématiquement en évidence. Il y a lieu de remarquer que le rôle de la CaBP intestinale a été remis en cause par Spencer et al. (1976). Même dans le cas où la CaBP serait présente dans le placenta, son rôle dans le mécanisme de transfert placentaire du calcium resterait à démontrer.

En revanche, l'activité Ca-ATPasique évolue parallèlement à l'intensité du flux placentaire de calcium, aussi bien dans des conditions nutritionnelles ef endocriniennes normales que chez les mères hypothyroïdiennes ou parathyroïdectomisées. On aurait pu s'attendre à une stimulation de la Ca-ATPase placentaire lorsque la mère est fortement hypocalcémique. En fait, la parathyroïdectomie semble avoir pour effet de réduire la taille des fœtus, ce qui correspond à un besoin moindre de calcium pour le fœitus, sans que soit modifiée de façon notable le nombre des points d'ossification dans le squelette des fœtus, par rapport à des témoins de même âge et plus lourds (résultats préliminaires).

Réunion Groupe Développement INRA/Productions animales Montpellier, 17-18 mai 1977.

Remerciements. - Ce travail a bénéficié d'une aide de la DGRST, contrat 7470042 . 


\section{Références}

KRETSINGER R. H., 1976. Evolution and function of calcium-binding proteins. Int. Rev. Cyfol., 46, 323-393.

PIC P., 1969. Etude de la régulation de la calcémie et de la phosphorémie foetales, chez le rat. Rôle des barathyroides foetales et du placenta. Thèse D. Sci., Paris.

SHAMI Y., RADDE I. C., 1971. Calcium-stimulated ATPase of guinea pig placenta. Biochim. biophys. ACta, 249, 345-352.

SPENCER R., CHARMAN M., WILSON P., LAWSON E., 1976. Vifamin D-stimulated intestinal calcium-absorption may not involve calcium-binding protein directly. Nature, 263, 161-162.

WASSERMAN R. H., TAYLOR A. N., 1966. Vitamin $D_{3}$-induced calcium-binding protein in chick intestinal mucosa. Science, 152, 791-793.

WHITTAKER V. P., BARKER L. A., 1972. 'In FRIED R. Methods of neurochemistry, 2, 1-52, M. Dekker Inc., New-York. 